\title{
Dysregulation of the PI3K/Akt signaling pathway affects cell cycle and apoptosis of side population cells in nasopharyngeal carcinoma
}

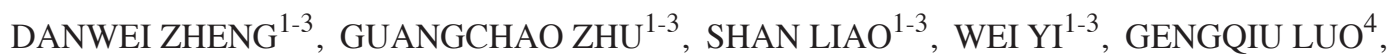 \\ JUNYU HE ${ }^{1-3}$, ZHEN PEI ${ }^{1-3}$, GUIYUAN $\mathrm{LI}^{1-3}$ and YANHONG ZHOU ${ }^{1-3}$
}

\begin{abstract}
${ }^{1}$ Medical Experimental Center, Hunan Provincial Tumor Hospital and The Affiliated Tumor Hospital of Xiangya School of Medicine, Central South University, Changsha, Hunan 410013; ${ }^{2}$ Molecular Genetics Laboratory, Key Laboratory of Carcinogenesis of Ministry of Health and Key Laboratory of Carcinogenesis and Cancer Invasion of Ministry of Education, Cancer Research Institute, Changsha, Hunan 410078; ${ }^{3}$ Hunan Key Laboratory of Nonresolving Inflammation and Cancer, Disease Genome Research Center, The Third Xiangya Hospital, Central South University, Changsha, Hunan 410013;

${ }^{4}$ Department of Pathology, Basic School of Medicine, Central South University, Changsha, Hunan 410008, P.R. China
\end{abstract}

Received July 24, 2014; Accepted April 8, 2015

DOI: $10.3892 / \mathrm{ol} .2015 .3218$

\begin{abstract}
Increasing evidence has suggested that certain types of cancer possess their own stem-like cells, and that one subset of these cells, termed the side population (SP), may have an important role in tumorigenesis and cancer therapy. However, the molecular mechanisms underlying the modulation of SP cells in nasopharyngeal carcinoma (NPC) have remained elusive. In the present study, it was hypothesized that dysregulation of the phosphatidylinositol-4,5-bisphosphate 3-kinase (PI3K)/Akt signaling pathway may influence SP and non-SP (NSP) phenotype. SP cells from the HK-1 NPC cell line were identified, and cancer stem cell markers were found to be highly expressed in SP cells compared with that of NSP cells. Freshly sorted SP cells demonstrated a significant increase in the proportion of cells in G0/G1 phase, while the majority of NSP cells were in the proliferative phase. Following $48 \mathrm{~h}$ of culture subsequent to cell sorting, the differences in cell cycle distribution between the SP and NSP cells converged. In addition, the apoptotic ratio of NSP cells was
\end{abstract}

Correspondence to: Dr Yanhong Zhou or Professor Guiyuan Li, Medical Experimental Center, Hunan Provincial Tumor Hospital and the Affiliated Tumor Hospital of Xiangya School of Medicine, Central South University, 286 Tongzipo Road, Changsha, Hunan 410013, P.R. China

E-mail: zhouyanhong@csu.edu.cn

E-mail: lgy@csu.edu.cn

Abbreviations: CSC, cancer stem cell; NPC, nasopharyngeal carcinoma; SP, side population; NSP, non side population; ABCG2, adenosine triphosphate-binding cassette transporter superfamily $G$ member 2; PI3K, phosphatidylinositol-4,5-bisphosphate 3-kinase; FCM, flow cytometry

Key words: nasopharyngeal carcinoma, side population cells, cell cycle, apoptosis, signal pathway higher than that of SP cells at $24 \mathrm{~h}$ following sorting, but had no significant differences $48 \mathrm{~h}$ following sorting. To elucidate the potential mechanism mediating the cell cycle and apoptosis in SP cells, the expression levels of key molecules in the PI3K/Akt signaling pathway were evaluated. PI3K and Akt

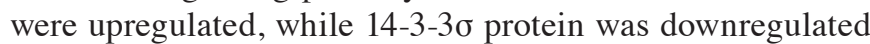
in SP cells when freshly sorted $(0 \mathrm{~h})$. However, there was no significant difference in the expression of these molecules between SP and NSP cells following $48 \mathrm{~h}$ of culture. These results suggested that dysregulation of the PI3K/Akt signaling pathway may be associated with the cell cycle and apoptosis of SP cells in NPC. However, further investigation is required to elucidate the detailed mechanisms underlying these effects.

\section{Introduction}

Increasing evidence has suggested that specific types of cancer may contain their own stem-like cells, known as cancer stem cells (CSCs), which have key roles in the initiation, maintenance and recurrence of tumors (1-3). In particular, attention has been paid to a subset of CSCs, termed the side population (SP), which was identified by flow cytometry. These SP cells are able to exclude the DNA binding dye, Hoechst 33342, and are highly enriched for stem cells in numerous types of tissue (4-6). SP cells have been isolated from multiple solid tumors, and studies have suggested that they may have significant roles in tumorigenesis and cancer therapy. A SP of cells in nasopharyngeal carcinoma (NPC) were found to exhibit characteristics of stem-like cancer cells (7-12). However, the molecular mechanisms underlying the modulation of these stem-like cell populations in NPC have remained elusive.

Cellular proliferation is a critical process underlying the growth, development and regeneration of eukaryotic organisms, and appropriate control of the cell cycle is required for the proliferation of normal cells $(13,14)$. Deregulation of the cell cycle is responsible for the aberrant cell proliferation characteristic of cancer, and the loss of cell cycle checkpoint control, 
which promotes genetic instability. The cell cycle machinery, which functions as an integration point for information transduced via upstream signaling networks, is a target for potential diagnostic and therapeutic interventions (13-15). Apoptosis is a physiological cell death process that has key functions in normal development, as well as in the pathophysiology of various diseases $(16,17)$. A balance between the expression of anti-apoptotic and pro-apoptotic factors underlies apoptosis; and this balance may be altered by certain extracellular signals. Significant alterations to this regulatory pathway may result in the development of various diseases, including autoimmune and neurodegenerative diseases, as well as certain types of cancer (16-19). The phosphatidylinositol-4,5-bisphosphate 3-kinase (PI3K)/Akt pathway is known to have key roles in cell proliferation, apoptosis and survival in various cell types (20). The PI3K/Akt signaling pathway has been shown to regulate metastasis in multiple cancer cells $(21,22)$.

In the present study, SP cells were identified in the HK-1 NPC cell line, and the SP and NSP cells within this population were sorted for analysis of the cell cycle and apoptosis at differential time-points. In addition, the expression levels of key molecules associated with the PI3K/Akt signaling pathway, including PI3K and Akt, were evaluated by western blotting at the corresponding time-points. The results of the present study may aid the elucidation of the involvement of dysregulation of the PI3K/Akt signaling pathway in cell cycle and apoptosis of SP cells in NPC.

\section{Materials and methods}

Cell culture. HK-1 human NPC cells, a highly differentiated NPC cell line, were provided by the Chinese University of Hong Kong (Hong Kong, China), and cultured in RPMI-1640 (Gibco Life Technologies, Grand Island, NY, USA) supplemented with $10 \%$ fetal bovine serum (FBS; Gibco Life Technologies), $100 \mathrm{U} / \mathrm{ml}$ penicillin and $100 \mu \mathrm{g} / \mathrm{ml}$ streptomycin (HyClone; GE Healthcare Life Sciences, Logan, UT, USA) at $37^{\circ} \mathrm{C}$ in a $5 \% \mathrm{CO}_{2}$ incubator.

Identifying and sorting of SP cells by flow cytometry (FCM). HK-1 cells were cultured in RPMI-1640 with 10\% FBS until they reached $\sim 70 \%$ confluence. The cells were trypsinized with $0.25 \%$ Trypsin (Sigma-Aldrich, St. Louis, MO, USA) at $37^{\circ} \mathrm{C}$ in a $5 \% \mathrm{CO}_{2}$ cell incubator. Following centrifugation at $500 \mathrm{x} \mathrm{g}$ for $5 \mathrm{~min}$ at room temperature, the single cell suspension was resuspended in prewarmed RPMI-1640 culture medium containing $2 \% \mathrm{FBS}$ at a concentration of $1 \times 10^{6}$ cells $/ \mathrm{ml}$. Hoechst 33342 (10 mg/ml; Biotium Inc., Hayward, CA, USA) was added at a final concentration of $5 \mu \mathrm{g} / \mathrm{ml}$ with or without $50 \mu \mathrm{mol} / 1$ verapamil (5 mmol/1; Sigma-Aldrich), an adenosine triphosphate binding cassette (ABC) transporter inhibitor, to determine whether the fluorescent efflux effect was altered. The cell suspensions were incubated in a $37^{\circ} \mathrm{C}$ circulating water bath for $90 \mathrm{~min}$ with gentle shaking every $15 \mathrm{~min}$. Subsequently, the cells were washed twice with pre-cooled phosphate-buffered saline (PBS; Solarbio Science and Technology Co., Ltd., Beijing, China), resuspended in iced PBS with 2\% FBS buffer and $1 \mu \mathrm{g} / \mathrm{ml}$ propidium iodide (PI; Sigma-Aldrich) was added to exclude dead cells. The entire protocol was performed in the dark. A MoFlo ${ }^{\mathrm{TM}}$ XDP high-performance cell sorter (Beckman
Coulter, Brea, CA, USA) was used for analysis of the SP profile and subsequent cell sorting. In the flow cytometry graphs, SP cells displayed a low Hoechst staining intensity. Finally, SP and NSP cells were sorted from the HK-1 cell line for further experiments. Data and images were acquired using Summit v.5.2 software (Beckman Coulter).

CSC marker assay in SP and NSP cells. The total expression and cell surface expression levels of various CSC markers were evaluated in sorted SP and NSP cells by flow cytometric analysis (MoFlo $\left.{ }^{\mathrm{TM}} \mathrm{XDP}\right)$. CSC cell surface marker expression was determined by washing freshly sorted SP and NSP cells with PBS, prior to incubation with the following fluorescent conjugated antibodies ( $5 \mu \mathrm{g} / 10^{5}-10^{7}$ cells): ABC superfamily $\mathrm{G}$ member 2 (ABCG2)-phycoerythrin (PE) (eBioscience, Inc., San Diego, CA, USA), CD133-PE [Miltenyi Biotec Technology \& Trading (Shanghai) Co., Ltd. Shanghai, China], CD34-electron-coupled dye [ECD (PE-Texas Red)] (Beckman Coulter), CD26-fluorescein isothiocyanate (FITC; Beckman Coulter), cytokeratin 14-FITC (Biological, Swampscott, MA, USA) for $1 \mathrm{~h}$ at $4^{\circ} \mathrm{C}$. PE mouse immunoglobulin $\mathrm{G}(\mathrm{IgG})_{2 \mathrm{~b}}$ isotype control (eBioscience, Inc.), mouse $\operatorname{IgG}_{2 b}$ isotype control FITC (eBioscience, Inc.) and mouse $\operatorname{IgG}_{2 \mathrm{~b}}$ isotype control ECD (eBioscience, Inc.) were used as negative controls for non-specific background signals.

To determine the total expression of these CSC markers, the sorted SP and NSP cells were fixed in $4 \%$ paraformaldehyde (Solarbio Science and Technology Co., Ltd.) for $30 \mathrm{~min}$, washed in PBS ( $3 \times 30 \mathrm{sec}$ ) and incubated with $0.1 \%$ Triton-X 100 (Solarbio Science and Technology Co., Ltd.) for 20 min. Subsequently, the cells were suspended in PBS and the corresponding aforementioned antibodies were added according to the manufacturer's instructions. Mouse $\operatorname{IgG}_{2 b}$ isotype control antibodies were used as the negative control. The results were analyzed by flow cytometry (MoFlo $\left.{ }^{\mathrm{TM}} \mathrm{XDP}\right)$.

$R N A$ isolation and reverse-transcription-quantitative polymerase chain reaction $(R T-q P C R)$ analysis. Total RNA was extracted from the SP and NSP cells using an RNeasy ${ }^{\circledR}$ kit (Qiagen, Inc., Valencia, CA, USA) and complemetary (c)DNA synthesis was performed using the RevertAid First Strand cDNA Synthesis kit (CWBio, Beijing, China) according to the manufacturer's instructions. Subsequently, qPCR was conducted using the GoTaq qPCR master mix (Promega Corp., Madison, WI, USA). The primers used for RT-qPCR are presented in Table I. RT-qPCR was performed using the BIO-RAD CFK96TM Real-Time System (Bio-Rad Laboratories, Inc., Hercules, CA, USA). The data were analyzed with Bio-Rad CFK Manager 2.0 software (Bio-Rad Laboratories, Inc.). Messenger (m)RNA expression was assessed by evaluating the threshold cycle (CT) values. GAPDH was used as an internal control.

Flow cytometric analysis of the cell cycle. The SP and NSP cells of the HK-1 cell line were sorted by flow cytometry as previously described, and divided into two groups, respectively. One group was for analysis of the cell cycle of sorted SP and NSP cells $(0 \mathrm{~h})$. The other was for the analysis of the cell cycle of SP and NSP cells following culture in RPMI-1640 supplemented with $10 \%$ fetal bovine serum for 24 or $48 \mathrm{~h}$. Cells were harvested at 0, 24 and $48 \mathrm{~h}$ and fixed in $70 \%$ ethanol at 
Table I. Human-specific primer sequences used in the present study. To avoid false positive signals originating from DNA contamination, all human-specific polymerase chain reaction primers were designed with known amplicon size, and where possible flanking a region that contained a minimum of one intron.

\begin{tabular}{|c|c|c|}
\hline Target gene & Forward primer & Reverse primer \\
\hline ABCG2 & AGCTGCAAGGAAAGATCCAA & TGCCCATCACAACATCATCT \\
\hline CD133 & TTGTGGCAAATCACCAGGTA & TCAGATCTGTGAACGCCTTG \\
\hline CD34 & CAAGCCACCAGAGCTATTCC & TCCACCGTTTTCCGTGTAAT \\
\hline $\mathrm{CD} 26$ & CAAATTGAAGCAGCCAGACA & CACACTTGAACACGCCACTT \\
\hline CK14 & TTCTGAACGAGATGCGTGAC & GCAGCTCAATCTCCAGGTTC \\
\hline
\end{tabular}

$4^{\circ} \mathrm{C}$. The cells were then washed with cold PBS and stained with PI in working solution $(0.5 \mathrm{mg} / \mathrm{ml} \mathrm{RNase}$ and $0.1 \mathrm{mg} / \mathrm{ml}$ PI in PBS). The cell cycle distribution was determined by flow cytometric analysis using a MoFlo ${ }^{\mathrm{TM}}$ XDP High-Performance Cell Sorter (Beckman Coulter) and the data were analyzed using Summit v.5.2 software.

Flow cytometric analysis of apoptosis. The SP and NSP cells of the HK-1 cell line were sorted by flow cytometry. The sorted cells were cultured in RPMI-1640 supplemented with $10 \%$ FBS for 24 or $48 \mathrm{~h}$, prior to harvest. The cell apoptosis ratio was analyzed using an Alexa Fluor ${ }^{\circledR} 488$ Annexin V/Dead Cell Apoptosis kit (Invitrogen Life Technologies, Carlsbad, CA, USA). Briefly, $5 \times 10^{5}$ cells were stained with Annexin V-FITC $(5 \mu \mathrm{l})$ and $100 \mu \mathrm{g} / \mathrm{ml} \mathrm{PI}(1 \mu \mathrm{l})$ in $100 \mu \mathrm{l}$ binding buffer and incubated at room temperature for $15 \mathrm{~min}$ in the dark. Subsequently, $400 \mu \mathrm{l}$ of binding buffer was added and mixed gently, and the stained cells were analyzed using a MoFlo ${ }^{\mathrm{TM}}$ XDP flow cytometer. The data were evaluated using Summit v.5.2 software.

Western blot analysis. SP and NSP cells at 0 and $48 \mathrm{~h}$ following sorting, were lysed in radioimmunoprecipitation buffer (CWBio, Beijing, China) and total protein concentration was determined using a Pierce ${ }^{\circledR}$ BCA Protein Assay kit (Thermo Fisher Scientific, Waltham, MA, USA). Extracts containing $50 \mu \mathrm{g}$ protein were separated with $10 \%$ SDS-PAGE and electroblotted onto nitrocellulose membranes (HyClone; GE Healthcare Life Sciences). The membranes were inhibited using Tris-buffered saline/Tween-20 (25 mM Tris- $\mathrm{HCl}, 150 \mathrm{mM} \mathrm{NaCl}$ and $0.05 \%$ Tween-20; pH 7.5; Solarbio Science and Technology Co., Ltd.) containing $5 \%$ non-fat milk followed by overnight incubation at $4^{\circ} \mathrm{C}$ with the following primary antibodies: rabbit anti-PI3K polyclonal antibody (catalog no. 4292; Cell Signaling Technology, Inc., Danvers, MA, USA; dilution, 1:500); rabbit anti-Akt polyclonal antibody, (catalog no. 9272; Cell Signaling Technology,

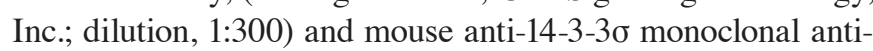
body (E-11; catalog no. sc-166473; Santa Cruz Biotechnology, Inc., Dallas, TX, USA). Following three washes, the membranes were incubated with horseradish peroxidase-conjugated mouse anti-rabbit (catalog no. sc-2491; Santa Cruz Biotechnology, Inc., Santa Cruz, CA, USA; dilution, 1:5,000) and goat anti-mouse (catalog no. sc-2039; Santa Cruz Biotechnology, Inc.; dilution, $1: 5,000) \mathrm{IgG}$ secondary antibodies for $1 \mathrm{~h}$ at room temperature and the signals were visualized using an enhanced chemiluminescence detection system (Universal Hood II; Bio-Rad
Laboratories, Inc.) with Image Lab ${ }^{\mathrm{TM}}$ software. Anti-GAPDH antibody (Santa Cruz Biotechnology, Inc.; 1:3,000) was used as a loading control.

Statistical analysis. Results were statistically analyzed by Student's unpaired t-test using SPSS 17.0 software (SPSS, Inc., Chicago, IL, USA). Data are presented as the mean \pm standard error of the mean. $\mathrm{P}<0.05$ was considered to indicate a statistically significant difference between values.

\section{Results}

Putative CSC markers are differentially expressed in SP and NSP cells of the HK-1 cell line. The SP fraction of the NPC cell line, HK-1, was determined using a flow cytometric SP discrimination assay. The percentage of SP cells was found to be $3.65 \pm 1.51 \%$. The proportion of SP cells was significantly blocked by verapamil (Fig. 1A and B). Subsequently, the MoFlo ${ }^{\mathrm{TM}} \mathrm{XDP}$ High-Performance Cell sorter was used to isolate the SP and NSP cells from the HK-1 cells and the cell surface expression levels of CSC markers were evaluated. The expression levels of putative CSC markers, including ABCG2, CD133, CD34 and CD26, were higher in SP cells compared with those of NSP cells $(\mathrm{P}<0.05)$. ABCG2 expression was higher in SP cells $(4.27 \%)$ than NSP cells $(0.41 \%)$. CD133 was 3.28 and $1.90 \%$ in SP and NSP cells, respectively. CD34 and CD26 exhibited analogous expression patterns. However, no differential expression of CK14 was detected between SP and NSP cells (Fig. 1C).

Protein and $m R N A$ expression levels of putative CSC markers differ between SP and NSP cells in NPC. Subsequently, the total protein expression levels of the putative CSC markers were examined in sorted SP and NSP cells by flow cytometry. The results demonstrated that CD133, CD34, and CK14 expression was higher in SP cells compared with that of NSP cells. Expression levels were 28.09, 28.17 and $11.89 \%$ in SP cells, and $4.82,24.90$ and $5.23 \%$ in NSP cells, respectively. The fraction of ABCG2 and CD26 expression was high in SP and NSP cells (Fig. 1D). In addition, the expression levels of these markers in SP and NSP cells were evaluated by RT-qPCR. It was demonstrated that there were significant differences in the mRNA expression levels of ABCG2, CD26, and CK14 between SP and NSP cells of the HK-1 cell line $(\mathrm{P}<0.05)$. However, there was no significant difference in the expression of CD133 and CD34 (Fig. 1E). In SP and NSP cells, the mRNA and protein expression levels of CSC markers, including CD133 and CD34, is not consistent. 

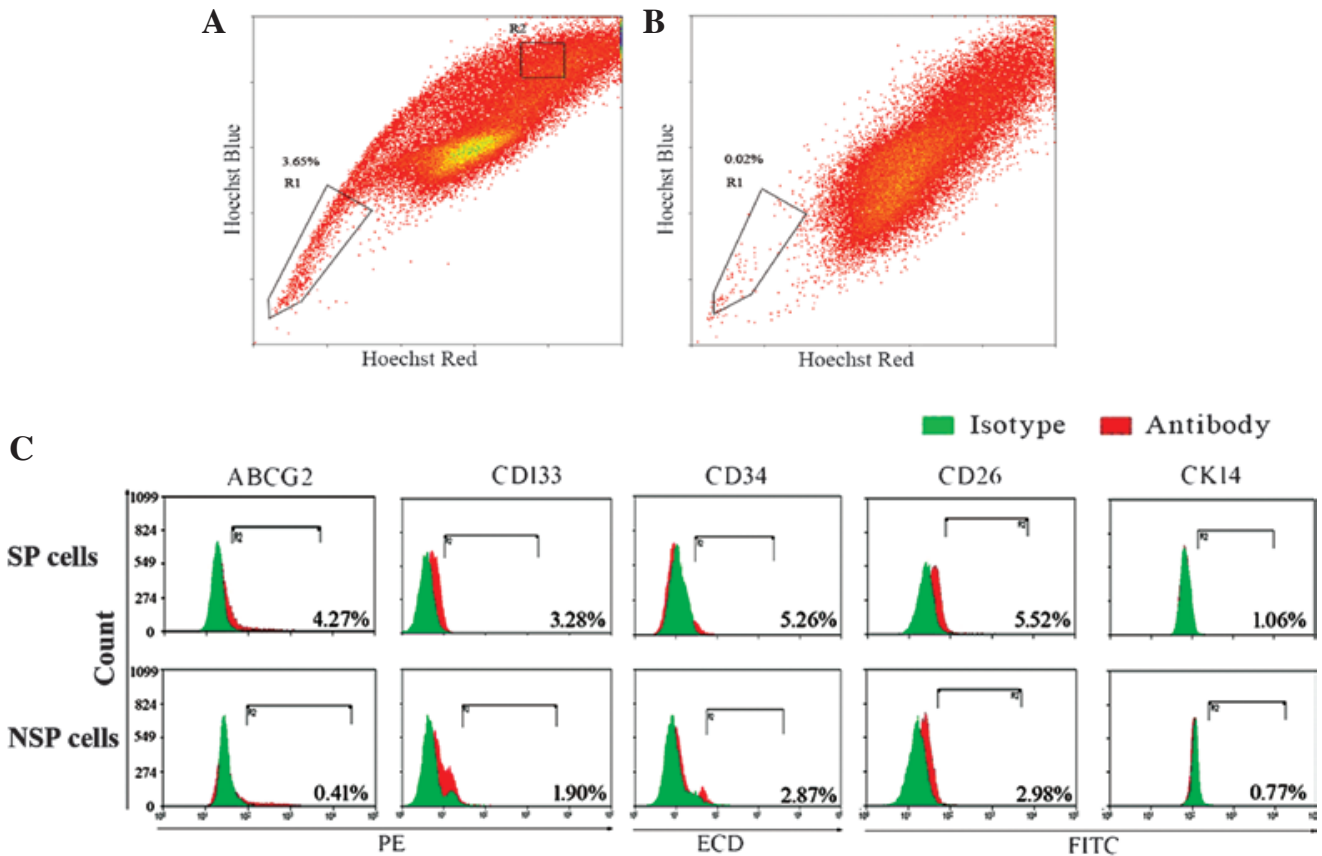

D
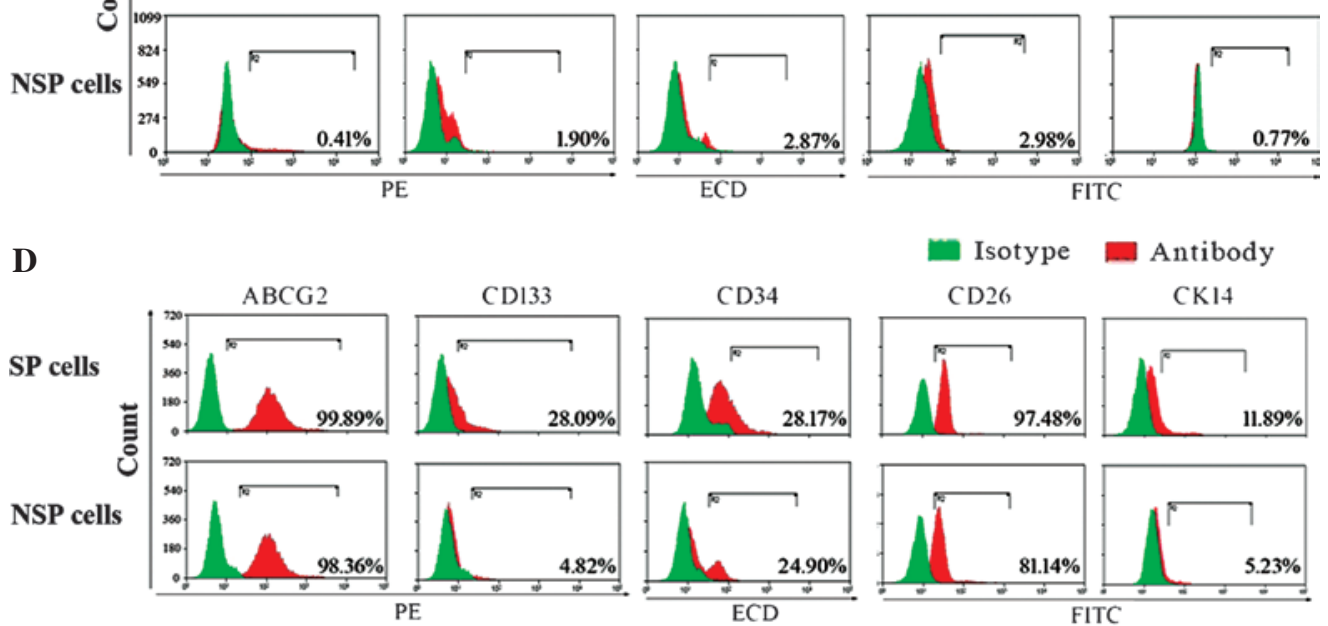

FITC
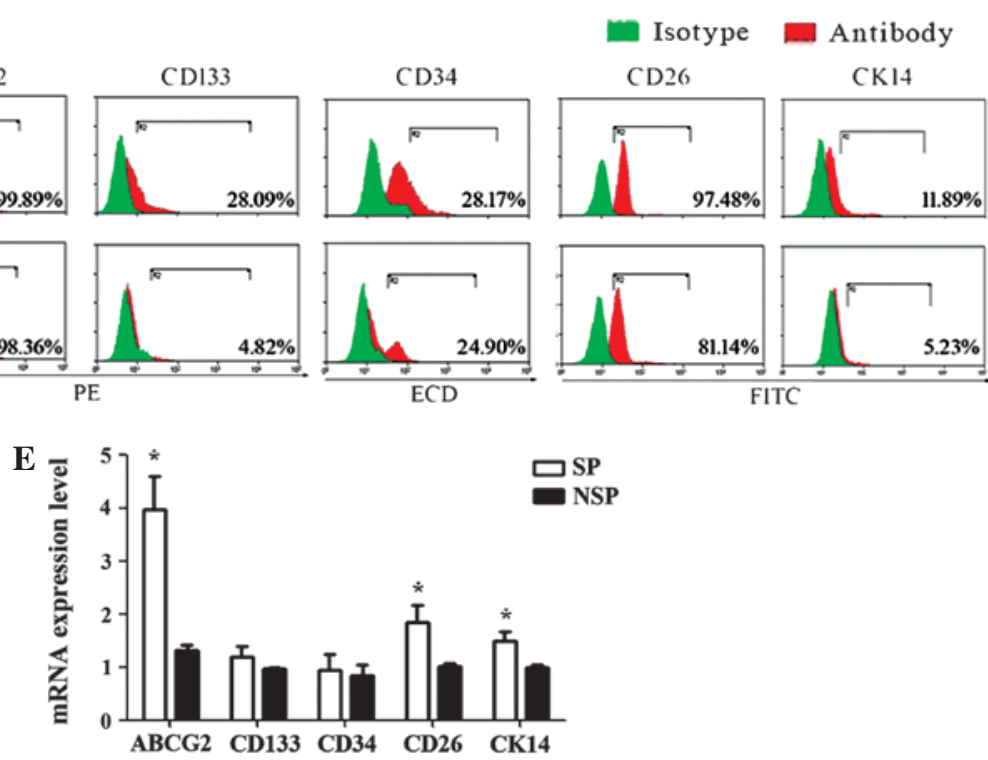

Figure 1. Human nasopharyngeal carcinoma cell lines contain a fraction of SP cells and CSC-associated markers are differentially expressed in SP and NSP cells. (A) Scatter-blot analysis of HK-1 cells stained with Hoechst 33342. (B) Scatter-blot analysis of HK-1 cells stained with Hoechst 33342 plus verapamil treatment. SP and NSP cells are indicated in boxes R1 and R2, respectively. (C) CSC marker, ABCG2, CD133, CD34, CD26 and CK14, expression on the cell surface of sorted SP and NSP cells was evaluated by flow cytometry. (D) Total expression levels of CSC markers, ABCG2, CD133, CD34, CD26 and CK14, in SP and NSP cells. Flow cytometric analysis revealed that CSCs markers were highly expressed in SP cells. (E) Relative mRNA expression levels of ABCG2, CD133, CD34, CD26 and CK14 in SP and NSP cells were determined by reverse transcription-quantitative polymerase chain reaction. Three independent experiments were performed. The results are shown as the mean \pm standard error and refer to freshly sorted cells $(0 \mathrm{~h})$. " $\mathrm{P}<0.05 \mathrm{vs}$. NSP cells. SP, side population; CSC, cancer stem cell; NSP, non-side population; mRNA, messenger RNA; PE, phycoerythrin; ECD, electron-coupled dye (PE-Texas Red); FITC, fluorescein isothiocyanate; ABCG2, adenosine triphosphate-binding cassette transporter superfamily G member 2.

Cell cycle distribution differs between freshly sorted $S P$ and NSP cells of the HK-1 cell line. Cell cycle progression was compared between SP and NSP cells 0,24 and $48 \mathrm{~h}$ following sorting by flow cytometric analysis. When cells were freshly sorted, SP cells revealed a significant increase in the proportion of cells in G0/G1 phase and a reduction in the percentage of cells in $\mathrm{S}$ phase (Fig. 2A). The percentages of $\mathrm{G} 0 / \mathrm{G} 1, \mathrm{~S}$ and $\mathrm{G} 2 / \mathrm{M}$ phases were $64,32.22$ and $3.78 \%$, respectively. By contrast, the majority of NSP cells were in the proliferative phase, and the percentages of cells in $\mathrm{G} 0 / \mathrm{G} 1$, $\mathrm{S}$ and $\mathrm{G} 2 / \mathrm{M}$ phases were $40.76,54.83$ and $4.41 \%$, respec- tively. Immediately following sorting, SP and NSP cells demonstrated significant differences in cell cycle distribution. However, following $24 \mathrm{~h}$ of culture, the differences in cell cycle distribution between SP and NSP cells were abrogated. The percentages of cells in G0/G1, S and G2/M phases in SP and NSP cells at $24 \mathrm{~h}$ were $44.09,19.55$ and 36.37 vs. $40.5 \%, 37.27 \%$ and $22.23 \%$, respectively (Fig. 2B). In accordance, the cell cycle distribution of SP and NSP cells $48 \mathrm{~h}$ following sorting also coincided. The percentages of cells in G0/G1, S and G2/M phase in SP and NSP cells at $48 \mathrm{~h}$ were $39.5 \%, 41.05 \%$ and $19.45 \%$ vs. $47.17 \%, 35.3 \%$ and 
A

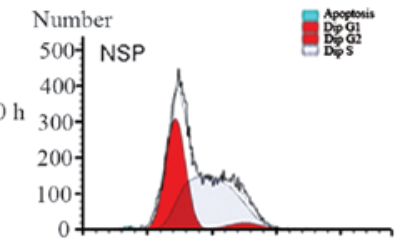

$\mathbf{B}$

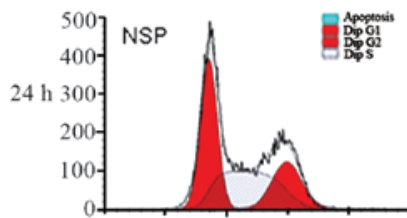

C

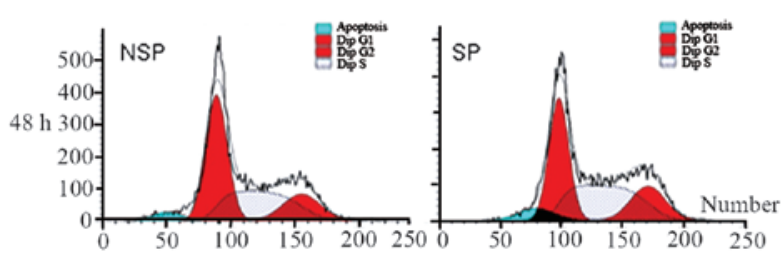

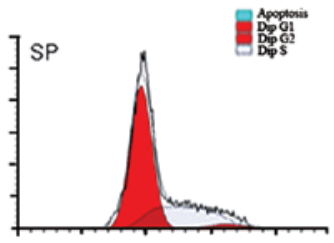

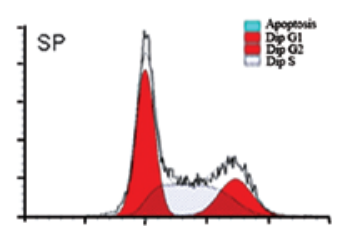

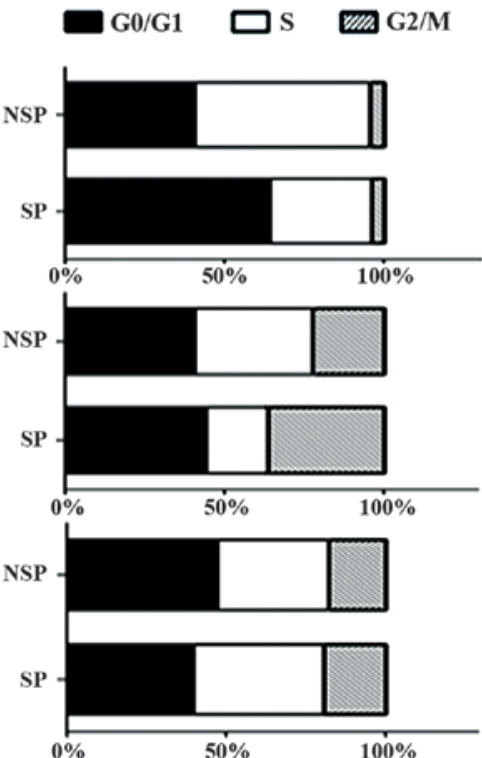

Figure 2. SP and NSP cells exhibited altered cell cycle profiles. Cell cycle distribution of SP and NSP cells was evaluated by flow cytometry (A) 0 , (B) 24 and (C) $48 \mathrm{~h}$ following cell sorting. SP, side population; NSP, non-side population.

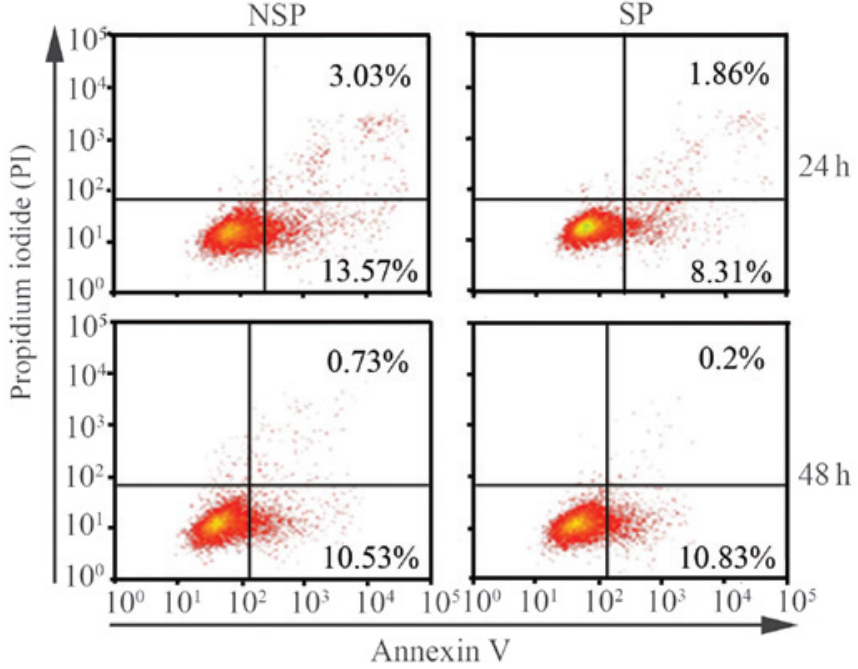

Figure 3. SP and NSP cells exhibited altered cell apoptosis profiles. Cell apoptotic analysis of SP and NSP cells by flow cytometry at 24 and $48 \mathrm{~h}$. Annexin V-positive/PI-negative cells indicated early apoptotic cells. Annexin V-positive/PI-positive cells indicated late apoptotic cells. The rate of cell apoptosis was calculated by early plus late apoptotic cells. SP, side population; NSP, non-side population; PI, propidium iodide.

$17.53 \%$, respectively (Fig. 2C). These results suggested that the differences in cell cycle distribution between SP and NSP cells converged with time.

The apoptotic ratio of NSP cells is higher than that of SP cells $24 \mathrm{~h}$ following sorting. Given that SP cells were found to be rich in CSCs markers compared with NSP cells, whether SP cells had a lower apoptotic ratio than NSP cells was determined. Annexin V-FITC and PI staining were used to analyze the percentage of apoptotic cells in SP and NSP cells at various time-points by flow cytometry. The apoptotic ratio of NSP cells was higher than that of SP cells $24 \mathrm{~h}$ following sorting, without any external stimuli to the

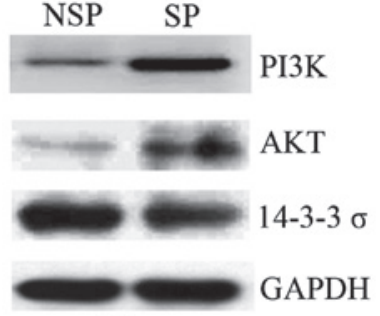

$0 \mathrm{~h}$

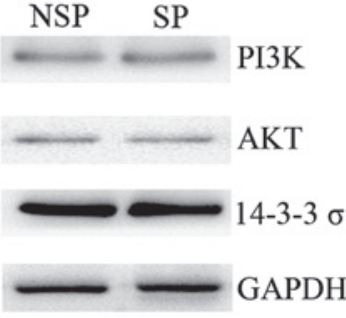

$48 \mathrm{~h}$
Figure 4. Western blot analysis of SP and NSP cells from HK-1 cell line for the indicated proteins at 0 and $48 \mathrm{~h}$ after cell sorting. GAPDH was used as the loading control. Expression levels of PI3K, AKT and 14-3-30 differed significantly at $0 \mathrm{~h}$ between SP and NSP cells. At $48 \mathrm{~h}$, no significant difference in expression was observed. SP, side population; NSP, non-side population; PI3K, phosphatidylinositol-4,5-bisphosphate 3-kinase.

cells. The apoptotic ratios of SP and NSP cells were 10.17 and $16.6 \%$, respectively. As observed in cell cycle distribution, no significant differences in the apoptotic proportion were detected between SP and NSP cells 48 h following sorting. The apoptotic ratios of SP and NSP cells at $48 \mathrm{~h}$ were 11.03 and $11.36 \%$, respectively (Fig. 3).

Dysregulation of The PI3K/Akt signaling pathway is dysregulated in SP cells of HK-1 cells immediately following sorting. To elucidate the potential mechanism underlying the mediation of the cell cycle and apoptosis in SP cells, the expression levels of key molecules associated with the PI3K/Akt signaling pathway were detected by western blot analysis. Immediately following cell sorting by flow cytometry $(0 \mathrm{~h}), \mathrm{PI} 3 \mathrm{~K}$ and Akt expression levels were upregulated in SP cells compared with those of NSP cells of the HK-1 cell line, whereas 14-3-3o protein expression was downregulated in SP cells. Once the sorted cells had been cultured for $48 \mathrm{~h}$, no significant difference in PI3K, Akt or 14-3-30 protein was detected between SP and NSP cells of HK-1 cells (Fig. 4). Combined with the 
aforementioned results of cell cycle and apoptosis analysis, it was hypothesized that dysregulation of the PI3K/Akt signaling pathway was associated with the alterations in the cell cycle and apoptosis of SP cells in NPC.

\section{Discussion}

NPC is an endemic disease with an incidence rate of 15-50/100,000 individuals in southern China and Southeast Asia, and represents one of the most significant public health issues in these regions (23). Although studies regarding the tumorigenesis of NPC have previously been published (24-29), the molecular basis for NPC is not fully understood. Recent studies have demonstrated that CSCs have a significant role in the pathophisiology of head and neck squamous cell carcinomas (30-32). In particular, research has focused on a specific subset of CSCs, termed SP cells, which were identified by FCM. Therefore, elucidation of the molecular mechanisms of SP in NPC is urgently required for the improvement of clinical diagnosis and therapy.

In the present study, the fraction of SP cells in the HK-1 NPC cell line, was found to be $3.65 \pm 1.51 \%$. The proportion of SP cells in this cell line was significantly decreased following verapamil treatment. SP cells expressed high levels of CSC markers compared with those of NSP cells. For example, ABCG2 expression was higher in SP cells (4.27\%) than in NSP cells $(0.41 \%)$, and CD133 expression was 3.28 and $1.90 \%$ in SP and NSP cells, respectively. Wang et al (7) revealed that $\mathrm{SP}$ cells represented $\sim 2.6 \%$ of the total cells in the NPC cell line, CNE-2. Another four human NPC cell lines, C-666-1, SUNE-1, HONE-1 and CNE-1, were also found to contain small subpopulations of SP cells and their proportions were $0.1,6.8,1.8$ and $0.7 \%$, respectively. Certain putative CSC markers are highly expressed in SP cells (7-9), and the results of these studies corroborate the results presented in the present study.

In order to reveal the characteristics of the cell cycle and apoptosis in SP cells, the cells were evaluated at differential time-points following sorting $(0,24$ or $48 \mathrm{~h})$. The results of the present study revealed that freshly sorted SP cells demonstrated a significant increase in the number of cells in G0/G1 phase. However, following 48 h of culture, differences in cell cycle distribution between SP and NSP cells were abrogated. In addition, the apoptotic ratio of NSP cells was higher than that of SP cells $24 \mathrm{~h}$ following sorting, whereas no significant differences were detected following $48 \mathrm{~h}$ of culture. We hypothesize that culturing the SP and NSP cells in complete medium after sorting may have caused the SP cells to differentiate, subsequently losing their stem cell properties. Previous studies have revealed that normal and neoplastic stem cells obtained from neural and epithelial organs only exhibit initial tumor-specific properties when cultured in serum-free medium containing epidermal growth factor (EGF) and fibroblast growth factor (FGF)-2 (33-35). In addition, adherent cells expanded in Laminin-coated culture plates in serum free medium containing N2-supplement, EGF and basic FGF maintain initial tumor-specific properties (36). However, when the cells were cultured in traditional complete medium, stem cells differentiated and lost their stem cell phenotype $(37,38)$. In contrast to embryonic stem cells, a characteristic feature of adult stem cells is their proliferative quiescence. It is widely accepted that this quiescent state is a functionally significant feature of adult stem cells (39-41).

To reveal the potential mechanisms underlying the cell cycle and apoptosis in SP cells, the expression levels of key molecules associated with the PI3K/Akt signaling pathway were detected. PI3K and Akt expression was upregulated, while 14-3-3 $\sigma$ protein expression was downregulated in freshly sorted SP cells ( 0 h). However, there was no significant difference in the expression of these molecules in SP and NSP cells following $48 \mathrm{~h}$ of culture. 14-3-3 $\sigma$, a potential tumor suppressor protein, is able to negatively regulate cell cycle progression by inducing G2-M phase arrest $(42,43)$. It has previously been demonstrated that $14-3-3 \sigma$ is transactivated by p53 in response to DNA damage and, in turn, interacts with p53 and positively regulates p53 activity (44). p53 is known to be involved in mediating the complex response to ionizing radiation, inducing irreversible growth arrest and apoptosis (45). The results of the present study are in accordance with those of previous reports.

In conclusion, the results of the present study suggested that dysregulation of the PI3K/Akt signaling pathway may be associated with mediation of the cell cycle and apoptosis of SP cells in NPC. However, elucidation of the detailed mechanisms underlying this process requires further study.

\section{Acknowledgements}

The present study was supported by the National Natural Science Foundation of China (no. 81272975), the Key Project of Hunan Provincial Natural Science Foundation (no. 12JJ2044), the Project of Hunan Provincial Natural Science Foundation (no. 12JJ3121), the Project of Hunan Provincial Development and Reform Commission and the Planned Science and Technology Project of Hunan Province (nos. 2010FJ3088 and 2012FJ2014).

\section{References}

1. Kristoffersen K, Villingshøj M, Poulsen HS and Stockhausen MT: Level of Notch activation determines the effect on growth and stem cell-like features in glioblastoma multiforme neurosphere cultures. Cancer Biol Ther 14: 625-637, 2013

2. Cetin I and Topcul M: Cancer stem cells in oncology. J BUON 17: 644-648, 2012.

3. Muñoz P, Iliou MS and Esteller M: Epigenetic alterations involved in cancer stem cell reprogramming. Mol Oncol 6: 620-636, 2012.

4. Mitsutake N, Iwao A, Nagai K, et al: Characterization of side population in thyroid cancer cell lines: Cancer stem-like cells are enriched partly but not exclusively. Endocrinology 148: 1797-1803, 2007.

5. Cao JX, Cui YX, Long ZJ, et al: Pluripotency-associated genes in human nasopharyngeal carcinoma CNE-2 cells are reactivated by a unique epigenetic sub-microenvironment. BMC Cancer 10: 68, 2010.

6. Hiraga $\mathrm{T}$, Ito $\mathrm{S}$ and Nakamura $\mathrm{H}$ : Side population in MDA-MB-231 human breast cancer cells exhibits cancer stem cell-like properties without higher bone-metastatic potential. Oncol Rep 25: 289-296, 2011.

7. Wang J, Guo LP, Chen LZ, Zeng YX and Lu SH: Identification of cancer stem cell-like side population cells in human nasopharyngeal carcinoma cell line. Cancer Res 67: 3716-3724, 2007.

8. Kong QL, Hu LJ, Cao JY, et al: Epstein-Barr virus-encoded LMP2A induces an epithelial-mesenchymal transition and increases the number of side population stem-like cancer cells in nasopharyngeal carcinoma. PLoS Pathog 6: e1000940, 2010. 
9. Liang Y, Zhong Z, Huang Y, Deng W, Cao J, Tsao G, Liu Q, Pei D, Kang $\mathrm{T}$ and Zeng YX: Stem-like cancer cells are inducible by increasing genomic instability in cancer cells. J Biol Chem 285: 4931-4940, 2010

10. Zhang HB, Ren CP, Yang XY, Wang L, Li H, Zhao M, Yang H and Yao KT: Identification of label-retaining cells in nasopharyngeal epithelia and nasopharyngeal carcinoma tissues. Histochem Cell Biol 127: 347-354, 2007.

11. Zhang Y, Peng J, Zhang H, Zhu Y, Wan L, Chen J, Chen X, Lin R, $\mathrm{Li} \mathrm{H}$, Mao X and Jin K: Notch1 signaling is activated in cells expressing embryonic stem cell proteins in human primary nasopharyngeal carcinoma. J Otolaryngol Head Neck Surg 39: 157-166, 2010.

12. Xia H, Cheung WK, Sze J,Lu G, Jiang S, Yao H, Bian XW, Poon WS, Kung HF and Lin MC: miR-200a regulates epithelial-mesenchyma to stem-like transition via ZEB2 and beta-catenin signaling. J Biol Chem 285: 36995-37004, 2010.

13. Diaz-Moralli S, Tarrado-Castellarnau M, Miranda A and Cascante M: Targeting cell cycle regulation in cancer therapy. Pharmacol Ther 138: 255-271, 2013.

14. Williams GH and Stoeber K: The cell cycle and cancer. J Pathol 226 352-364, 2012.

15. Aarts M, Linardopoulos S and Turner NC: Tumour selective targeting of cell cycle kinases for cancer treatment. Curr Opin Pharmacol 13: 529-535, 2013.

16. Sankari SL, Masthan KM, Babu NA, Bhattacharjee T and Elumalai M: Apoptosis in cancer - an update. Asian Pac J Cancer Prev 13: 4873-4878, 2012.

17. Zielinski RR, Eigl BJ and Chi KN: Targeting the apoptosis pathway in prostate cancer. Cancer J 19: 79-89, 2013.

18. Beesoo R, Neergheen-Bhujun V, Bhagooli R and Bahorun T: Apoptosis inducing lead compounds isolated from marine organisms of potential relevance in cancer treatment. Mutat Res Fundam Mol Mech Mutagen 768: 84-97, 2014.

19. Jia LT, Chen SY and Yang AG: Cancer gene therapy targeting cellular apoptosis machinery. Cancer Treat Rev 38: 868-876, 2012.

20. Qiao M, Sheng S and Pardee AB: Metastasis and AKT activation. Cell Cycle 7: 2991-2996, 2008.

21. Vivanco I and Sawyers CL: The phosphatidylinositol 3-kinase AKT pathway in human cancer. Nat Rev Cancer 2: 489-501, 2002

22. Wagner EF and Nebreda AR: Signal integration by JNK and p38 MAPK pathways in cancer development. Nat Rev Cancer 9: 537-549, 2009.

23. Ho JH: An epidemiologic and clinical study of nasopharyngeal carcinoma. Int J Radiat Oncol Biol Phys 4: 182-198, 1978

24. Zhou Y, Wang W, Zheng D, Peng S, Xiong W, Ma J, Zeng Z, Wu M, Zhou M, Xiang J, et al: Risk of nasopharyngeal carcinoma associated with polymorphic lactotransferrin haplotypes. Med Oncol 29: 1456-1462, 2012.

25. Zhou Y, Zeng Z, Zhang W, Xiong W, Wu M, Tan Y, Yi W, Xiao L, Li X, Huang C, et al: Lactotransferrin, a candidate tumor suppressor, deficient expression in human nasopharyngeal carcinoma and inhibits NPC cell proliferation by modulating the mitogen-activated protein kinase pathway. Int J Cancer 123: 2065-2072, 2008.

26. Zhou Y, Zeng Z, Zhang W, Xiong W, Li X, Zhang B, Yi W, Xiao L, $\mathrm{Wu} \mathrm{M}$, Shen $\mathrm{S}$, et al: Identification of candidate molecular markers of nasopharyngeal carcinoma by microarray analysis of subtracted cDNA libraries constructed by suppression subtractive hybridization. Eur J Cancer Prev 17: 561-571, 2008

27. Zeng Z, Zhou Y, Xiong W, Luo X, Zhang W, Li X, Fan S, Cao L, Tang $\mathrm{K}, \mathrm{Wu} \mathrm{M}$ and Li G: Analysis of gene expression identifies candidate molecular markers in nasopharyngeal carcinoma using microdissection and cDNA microarray. J Cancer Res Clin Oncol 133: 71-81, 2007.
28. Zeng ZY, Zhou YH, Zhang WL, Xiong W, Fan SQ, Li XL, Luo XM, Wu MH, Yang YX, Huang C, et al: Gene expression profiling of nasopharyngeal carcinoma reveals the abnormally regulated WNT signaling pathway. Hum Pathol 38: $120-133,2007$

29. Zeng Z, Zhou Y, Zhang W, Li X, Xiong W, Liu H, Fan S, Qian J, Wang L, Li Z, et al: Family-based association analysis validates chromosome $3 \mathrm{p} 21$ as a putative nasopharyngeal carcinoma susceptibility locus. Genet Med 8: 156-160, 2006.

30. Szafarowski T and Szczepanski MJ: Cancer stem cells in head and neck squamous cell carcinoma. Otolaryngol Pol 68: 105-111, 2014.

31. Qian X, Wagner S, Ma C, Coordes A, Gekeler J, Klussmann JP, Hummel M, Kaufmann AM and Albers AE: Prognostic significance of ALDH1A1-positive cancer stem cells in patients with locally advanced, metastasized head and neck squamous cell carcinoma. J Cancer Res Clin Oncol 140: 1151-1158, 2014.

32. Han J, Fujisawa T, Husain SR and Puri RK: Identification and characterization of cancer stem cells in human head and neck squamous cell carcinoma. BMC Cancer 14: 173, 2014.

33. Singh SK, Clarke ID, Terasaki M, Bonn VE, Hawkins C, Squire $\mathrm{J}$ and Dirks PB: Identification of a cancer stem cell in human brain tumors. Cancer Res 63: 5821-5828, 2003.

34. Ricci-Vitiani L, Lombardi DG, Pilozzi E, Biffoni M, Todaro M, Peschle $C$ and De Maria R: Identification and expansion of human colon-cancer-initiating cells. Nature 445: 111-115, 2007.

35. Wu A, Luo W, Zhang Q, Yang Z, Zhang G, Li S and Yao K: Aldehyde dehydrogenase 1, a functional marker for identifying cancer stem cells in human nasopharyngeal carcinoma. Cancer Lett 330: 181-189, 2013.

36. Pollard SM, Yoshikawa K, Clarke ID, Danovi D, Stricker S, Russell R, Bayani J, Head R, Lee M, Bernstein M, et al: Glioma stem cell lines expanded in adherent culture have tumor-specific phenotypes and are suitable for chemical and genetic Screens. Cell Stem Cell 4: 568-580, 2009.

37. Han ME, Jeon TY, Hwang SH, Lee YS, Kim HJ, Shim HE, Yoon S, Baek SY, Kim BS, Kang CD and Oh SO: Cancer spheres from gastric cancer patients provide an ideal model system for cancer stem cell research. Cell Mol Life Sci 68: 3589-3605, 2011

38. Singh S, Trevino J, Bora-Singhal N, Coppola D, Haura E, Altiok S and Chellappan SP: EGFR/Src/Akt signaling modulates Sox 2 expression and self-renewal of stem-like sidepopulation cells in non-small cell lung cancer. Mol Cancer 11: $73,2012$.

39. Orford KW and Scadden DT: Deconstructing stem cell self-renewal: Genetic insights into cell-cycle regulation. Nat Rev Genet 9: 115-128, 2008 .

40. Horsley V, Aliprantis AO, Polak L, Glimcher LH and Fuchs E: NFATc1 balances quiescence and proliferation of skin stem cells. Cell 132: 299-310, 2008.

41. Malumbres M: Physiological relevance of cell cycle kinases. Physiol Rev 91: 973-1007, 2011.

42. Mhawech P: 14-3-3 proteins - an update. Cell Res 15: 228-236, 2005

43. Laronga C, Yang HY, Neal C and Lee MH: Association of the cyclin-dependent kinases and 14-3-30 negatively regulates cell cycle progression. J Biol Chem 275: 23106-23112, 2000.

44. Yang HY, Wen YY, Chen CH, Lozano G and Lee MH: 14-3-3o positively regulates $\mathrm{p} 53$ and suppresses tumor growth. Mol Cell Biol 23: 7096-7107, 2003.

45. Gudkov AV and Komarova EA: The role of p53 in determining sensitivity to radiotherapy. Nat Rev Cancer 3: 117-129, 2003. 